\title{
Heterodyne detector for measuring the characteristic of elliptically polarized microwaves
}

\author{
Leipold, Frank; Nielsen, Stefan Kragh; Michelsen, Susanne
}

Published in:

Review of Scientific Instruments

Link to article, DOI:

$10.1063 / 1.2937651$

Publication date:

2008

Document Version

Publisher's PDF, also known as Version of record

Link back to DTU Orbit

Citation (APA):

Leipold, F., Nielsen, S. K., \& Michelsen, S. (2008). Heterodyne detector for measuring the characteristic of elliptically polarized microwaves. Review of Scientific Instruments, 79(6), 065103.

https://doi.org/10.1063/1.2937651

\section{General rights}

Copyright and moral rights for the publications made accessible in the public portal are retained by the authors and/or other copyright owners and it is a condition of accessing publications that users recognise and abide by the legal requirements associated with these rights.

- Users may download and print one copy of any publication from the public portal for the purpose of private study or research.

- You may not further distribute the material or use it for any profit-making activity or commercial gain

- You may freely distribute the URL identifying the publication in the public portal

If you believe that this document breaches copyright please contact us providing details, and we will remove access to the work immediately and investigate your claim. 


\title{
Heterodyne detector for measuring the characteristic of elliptically polarized microwaves
}

\author{
Frank Leipold, Stefan Nielsen, and Susanne Michelsen \\ Association EURATOM, Risф National Laboratory, Technical University of Denmark, \\ OPL-128, Frederiksborgvej 399, 4000 Roskilde, Denmark
}

(Received 28 January 2008; accepted 6 May 2008; published online 4 June 2008)

\begin{abstract}
In the present paper, a device is introduced, which is capable of determining the three characteristic parameters of elliptically polarized light (ellipticity, angle of ellipticity, and direction of rotation) for microwave radiation at a frequency of $110 \mathrm{GHz}$. The device consists of two perpendicular orientated pickup waveguides. A heterodyne technique mixes the microwave frequency down to frequencies on the order of $200 \mathrm{MHz}$. An oscilloscope is used to determine the relative amplitudes of the electrical fields and the phase shift in between, from which the three characteristic parameters can be calculated. Results from measured and calculated wave characteristics of an elliptically polarized $110 \mathrm{GHz}$ microwave beam for plasma heating launched into the TEXTOR-tokamak experiment are presented. Measurement and calculation are in good agreement. (C) 2008 American Institute of Physics. [DOI: 10.1063/1.2937651]
\end{abstract}

\section{INTRODUCTION}

Elliptically polarized microwaves in the range above $100 \mathrm{GHz}$ play an important role for plasma heating, current drive, and scattering of radiation in tokamak plasma experiments. ${ }^{1,2}$ High power microwave sources (gyrotrons) are used for these applications. A gyrotron generates linearly polarized waves. In order to transform a linearly polarized wave into an elliptically polarized wave, polarizer plates are employed. $^{3,4}$ A polarizer plate is a mirror with parallel grooves on the surface. In a simplified picture, an electrical field component which is oriented parallel to the grooves is reflected on top of the grooves. The electrical field component, which is oriented perpendicular to the grooves is reflected at the bottom of the grooves. This causes a phase shift with respect to the electrical field component reflected on top of the grooves. A detailed description of the diffraction of electromagnetic waves with perfectly electrical conducting rectangular grooved grating is described in Ref. 5. The phase shift depends on the design of the polarizer plate. The groove depths and width are dependent on the used frequency and the desired phase shift. The superposition of both electrical field components provides, in general, elliptically polarized waves.

Characterization of elliptically polarized microwaves in the frequency range of $110 \mathrm{GHz}$ is important, e.g., for the diagnostic technique of collective Thomson scattering. 6,7 This technique uses a probing microwave beam launched into the plasma and detects scattered light leaving the plasma. Probing beam and scattered signal are generally elliptically polarized. In the case of the probing beam, the required elliptically polarized light is obtained by transforming linearly polarized waves from a gyrotron by means of a set of two polarizer plates. The elliptically polarized scattered signal leaving the plasma is transformed by means of polarizer plates into linearly polarized light which can be accepted by the receiver. Theoretically, the elliptically polarized wave characteristics obtained by polarizer plates in the transmission line can be calculated from the orientation of the polarizer plates (when their characteristics are known). However, it is desirable to verify the calculated results by measurements.

Elliptically polarized light is obtained, when two perpendicularly orientated linearly polarized waves with a phase shift are superimposed. The propagation directions of both waves is the $z$ direction and $E_{x}$ and $E_{y}$ are the electrical field components in the $x$ and $y$ directions, respectively. Figure 1 shows an example of two waves $\left(E_{x}\right.$ and $\left.E_{y}\right)$ with a phase shift of $50^{\circ}$. The ratio of the amplitudes $E_{y} / E_{x}$ is 0.7 . Figure 2 shows the projection of the electrical field in the $x-y$ plane. The strength of the electrical field is represented by the distance between the origin and the boundary of the ellipse. The electrical field vector rotates, as described in the picture.

The characteristic of elliptically polarized light can be described by three parameters: ellipticity $(\varepsilon)$, angle of ellipticity $(\Theta)$, and direction of rotation $(\rho) .^{8-10} \varepsilon$ is the ratio of the minor axis to the major axis of the ellipse describing the electrical field in the $x-y$ plane. It varies between 0 (linearly polarized light) and 1 (circularly polarized light). $\Theta$ is the angle between the major axis of the ellipse and the $x$ axis in turning direction toward the $y$ axis (mathematically positive direction). It varies between $0^{\circ}$ and $180^{\circ} . \rho$ can either be left-hand polarized or right-hand polarized. The electrical field vector of a right-hand polarized wave propagating in the positive $z$ direction in a right handed coordinate system rotates in the mathematically negative direction when projected into the $x-y$ plane. The transformation from the parameters, $E_{x}, E_{y}$, and phase shift between $E_{x}$ and $E_{y}$ to the characteristic parameters $\Theta, \varepsilon$, and $\rho$ is described in detail in Ref. 8. In the example above (Figs. 1 and 2), the angle of ellipticity $\Theta$ is $30.2^{\circ}$, the ellipticity $\varepsilon$ is 0.42 , and the rotation $\rho$ is right-hand polarized.

$\varepsilon$ and $\Theta$ of elliptically polarized waves can be obtained 


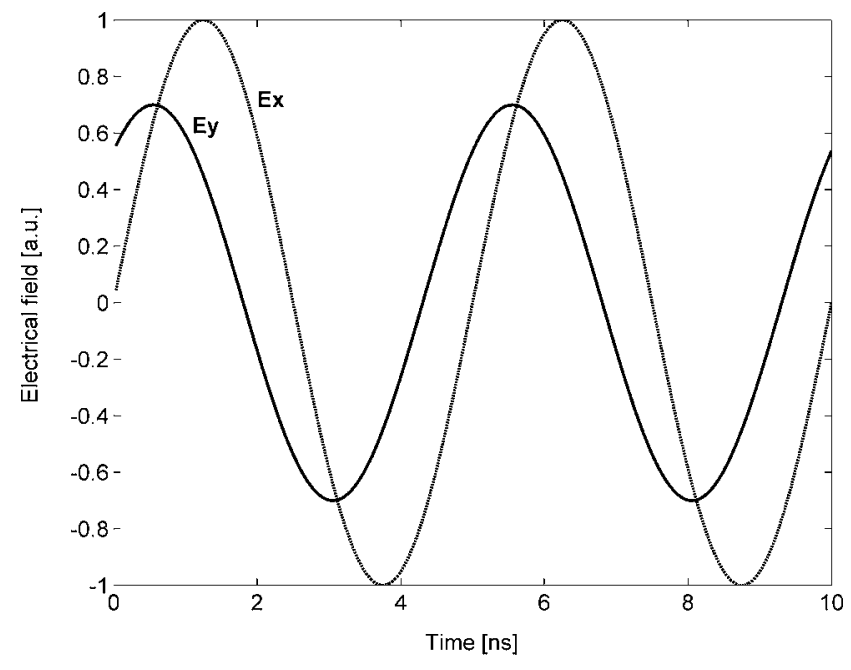

FIG. 1. The relative electrical field strength in the directions of $x\left(E_{x}\right)$ and $y$ $\left(E_{y}\right)$. In this example, $E_{y}$ leads $E_{x}$ by $50^{\circ}$.

by detecting the light intensity in various orientations of a detector diode in the $x-y$ plane. This technique does not require a complex system. However, since the phase difference of the measured electrical field in various directions cannot be detected, the rotation of the elliptically polarized wave cannot be obtained in this approach. Furthermore, several measurements in various orientations of the detector are required, which is a time consuming procedure when a complete mapping of a transmission line containing several polarizers is needed. The authors in Ref. 11 used a rotating birefringent plate in front of a detector in order to characterize an elliptically polarized wave. This technique provides all three parameters of the elliptically polarized wave. The authors in Ref. 12 introduced a method for characterization of an elliptically polarized wave, in which the detected $x$ and $y$ components of the electrical field are added and subtracted in a "Magic T." Sum and difference signals are recorded. In a

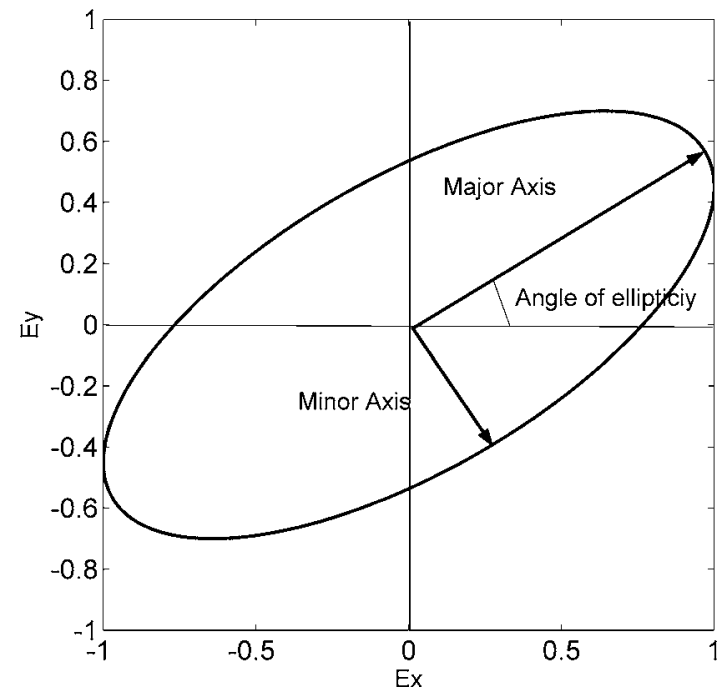

FIG. 2. Projection of the electrical field vector in the $x-y$ plane. The electrical field vector rotates in the mathematically negative direction (from the positive $x$ axis toward the negative $y$ axis) resulting in a right-hand elliptically polarized wave in a right handed coordinate system ( $z$ axis pointing toward the observer).

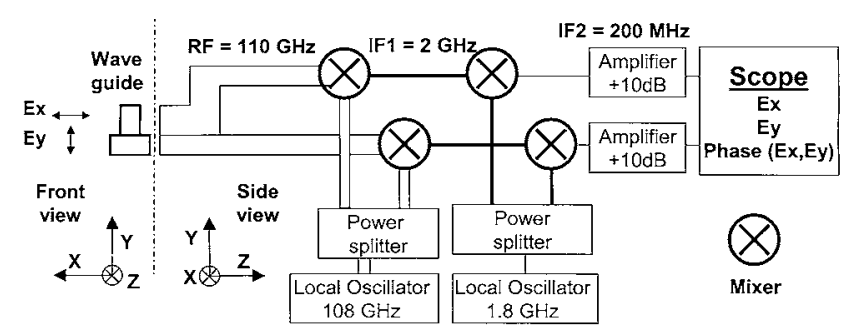

FIG. 3. Schematic of the detector for elliptically polarized light.

second step, the $y$ component of the electrical field is phase shifted by $90^{\circ}$ degrees, and again, sum and difference signals are recorded. These four values allow the characterization of the elliptically polarized waves.

The present article reports about a two-channel heterodyne detection system which is built in order to fully characterize elliptically polarized waves instantaneously without the use of movable mechanical parts. Principles of heterodyne techniques can be found in Refs. 13 and 14 .

\section{DEVICE SETUP}

The intensity of microwave radiation can easily be measured by detector diodes or Schottky diodes, but this technique does not provide information about the phase between the electrical field components detected in the two channels. In order to detect the phase difference between the fields in the two channels, a more sophisticated technique is required.

For the description and operation of this device, which is sketched in Fig. 3, a right-handed coordinate system is considered, in which the wave propagates in the positive $z$ direction. The electrical field of the wave is perpendicular to the axis of propagation and has components in the $x$ and $y$ directions which are referred to as $E_{x}$ and $E_{y}$, respectively. The axis of the detector is aligned parallel to the $z$ axis. The detector has two channels, which can detect the electrical field in two orthogonal orientations in the $x-y$ plane simultaneously. In order to obtain the three characteristic parameters of the elliptically polarized wave, the amplitudes of the signal in each channel and the phase between the two measured signals need to be known. The measured signal in each chan-

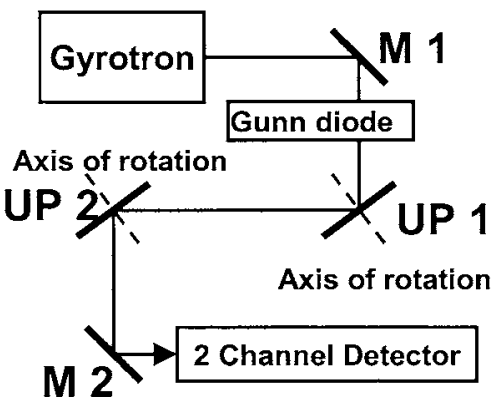

FIG. 4. Schematic of the transmission line in the gyrotron box at the TEXTOR-tokamak experiment. The Gunn diode is used for the measurement instead of the gyrotron as the gyrotron is too powerful and would destroy the detector. The mirror M2 does not belong to the gyrotron box. It was only used for convenience in the measurement. The change in rotation of the elliptically polarized wave leaving the gyrotron box due to reflection on M2 was taken into account. 

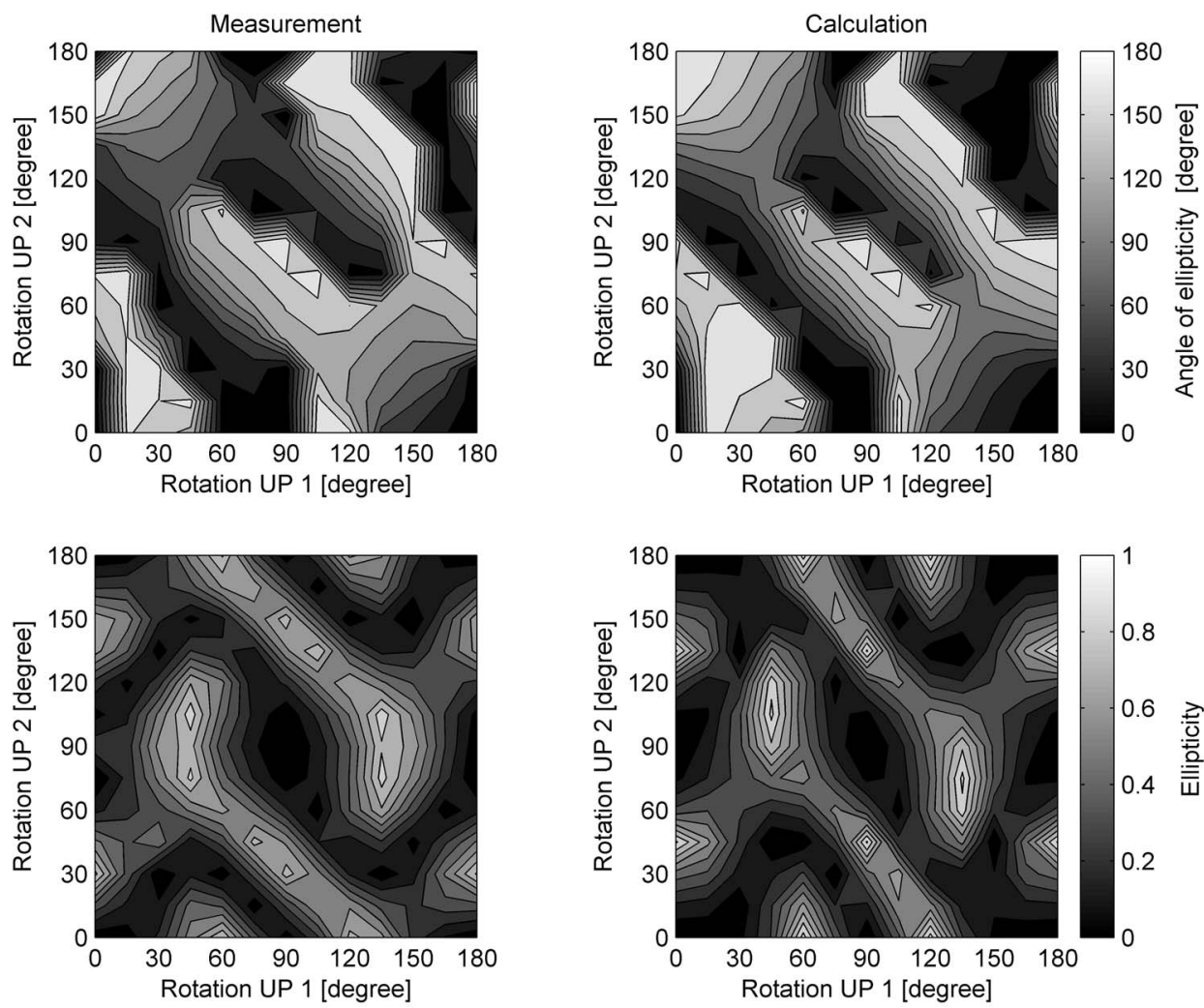

FIG. 5. Measured and calculated characteristics of the wave of the TEXTOR gyrotron vs the two polarizer settings at the exit of the gyrotron box. The resolution is $15^{\circ}$.
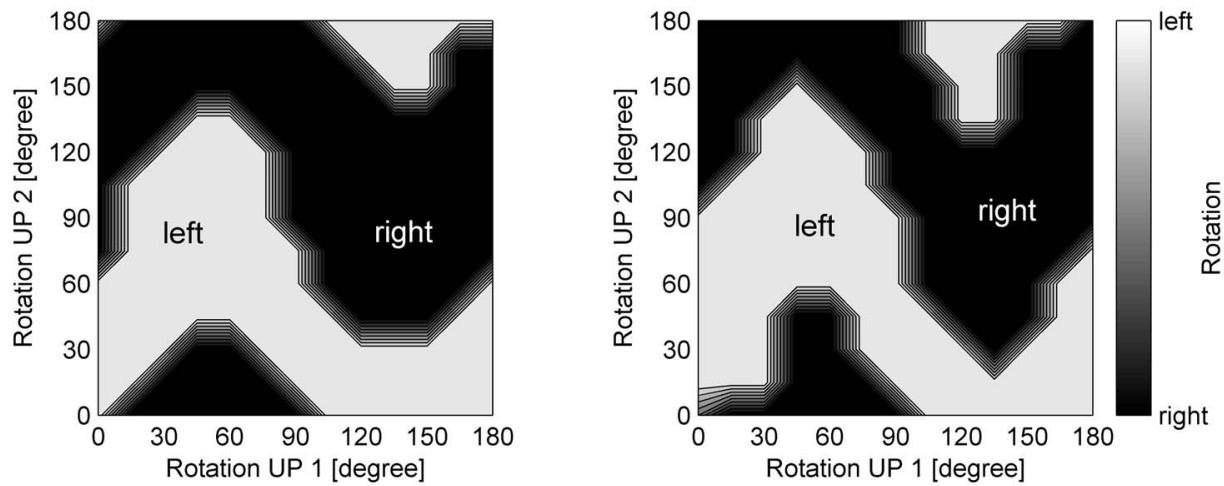

nel is mixed with a frequency close to $110 \mathrm{GHz}$. The goal of this mixing is to obtain a first intermediate frequency (IF1), which can be handled electrically. In order to conserve the phase information between the two channels, the mixers in both channels must be fed with the same local oscillator. Since IF1 is given by the available local oscillator, it still can be too high (several gigahertz) to be detected by an oscilloscope but it is sufficiently low to be processed electrically. In this case, a second mixing stage is employed, where a tunable frequency generator is used as local oscillator. Again, in order to conserve the phase information, both mixers must be fed with the same local oscillator. This means that the second intermediate frequency (IF2) can be chosen. A typical value for IF2 is $200 \mathrm{MHz}$. A signal of this frequency can be analyzed by a fast oscilloscope. From the amplitudes of both signals and the phase in between, the three characteristic parameters can be calculated.

\section{EXPERIMENTAL SETUP}

Using this measuring technique, the elliptically polarized wave emitted from the gyrotron box at the TEXTOR- tokamak experiment for various polarizer settings has been mapped. Figure 4 shows the quasioptical transmission in the gyrotron box before the microwaves are transmitted toward the plasma. The complete transmission line of the electron cyclotron resonance heating at the TEXTOR-tokamak experiment is detailed in Refs. 15 and 16. Horizontal linearly polarized light at a frequency of $110 \mathrm{GHz}$ is emitted from the gyrotron. It is reflected at the mirror M1 to the polarizer UP1 and from UP1 to the polarizer UP2. After the polarizer UP2, the beam leaves the gyrotron box and is guided through a transmission line into the tokamak. The polarizers UP1 and UP2 are identical. Depending on the settings of UP1 and UP2, all possible characteristics of elliptically polarized light can be obtained. The gyrotron cannot be used as microwave source for the characterization of the polarizer plates, as the high intensity would destroy the detector. For the measurements, a Gunn diode with the same frequency as the gyrotron was placed in front of polarizer UP1 with the polarization orientation parallel to the polarization orientation of the gyrotron.

For the mapping of the polarizer settings versus the char- 
acteristics of the polarization of the beam, our detector was placed at the exit of the gyrotron box (Fig. 4). Prior to the measurements, a calibration of the detection device needs to be done. The calibration consists of an amplitude calibration for each channel and a phase calibration between the two channels.

If both polarizers are tuned to their zero position, the grooves are parallel to the orientation of the electrical field. This means that the wave, seen at the detection device, is also linearly polarized. The orientation of the device is chosen so that the major axes of the device do not coincide with the direction of the linearly polarized electrical field. Each channel sees a component of the linearly polarized electrical field, which must have the same phase. The detected phase difference between both channels is due to the different transmission lengths in both channels. The detected phase difference is used for phase calibration.

For the intensity calibration, the polarizer plates were varied until one channel in the device did not show a signal. Therefore, all intensity goes to the other channel. The measured signal served as calibration. The same was done for the other channel.

\section{RESULTS}

For the mapping, both polarizers were rotated from $0^{\circ}$ to $180^{\circ}$ in $15^{\circ}$ increments, for which measurements were taken. The three characteristic parameters were obtained from the amplitudes of the signal of each channel and the phase shift between the signals of both channels. The results are plotted in Fig. 5, left column. The measurements were performed with a Gunn diode and a horn antenna. The Gunn diode had a power on the order of $20 \mathrm{~mW}$. The beam of the Gunn diode was expanded, so only a fraction of the power (less than $1 \mathrm{~mW}$ ) was received by the pickup waveguides. The oscilloscope recorded a signal on the order of $10 \mathrm{mV}$. The accuracy of the detector is basically given by the accuracy of the measurement of the voltage and the phase between both signals. However, two special cases shall be considered in more detail. First, when the detected light is almost linearly polarized, the phase shift between the signal is $0\left(180^{\circ}\right)$. Fluctuations of the phase measurement around $0\left(180^{\circ}\right)$ results in a change from left-hand polarized light to right-hand polarized light. In practice, this is not relevant, since the light pattern projected into the $x-y$ plane is an extremely elongated ellipse ( $\varepsilon$ close to 0 ). Second, when the detected light is circularly polarized (a circle in the $x$-y-plane projection), the angle of ellipticity is not defined, as minor and major axes are identical. Slight fluctuations in the measurement cause a change from the circle to an ellipse whereas the direction of the major axis and consequently, the angle of ellipticity cannot be predicted. In order to obtain an estimate of the accuracy of the measurements, the values between polarizer settings of $0^{\circ}$ and $180^{\circ}$ have been compared and the same results were expected. For a wave with an ellipticity $\varepsilon$ greater than 0.1 , the measurement was reproduced within an error of $3 \%$. The angle of ellipticity was accurate within $4^{\circ}$.
The manufacturer of the gyrotron provided a specification of each polarizer in the gyrotron box, in which the phase shift between light reflected on top of the grooves and light reflected at the bottom of the grooves was specified versus the polarizer rotation angle. Based on this characteristic, the characteristic of the light expected at the exit of the gyrotron box was calculated. The calculations for the three characteristic parameters are shown in Fig. 5, right column. The calculations were done with the same resolution $\left(15^{\circ}\right.$ steps $)$ as the measurements for better comparison.

\section{CONCLUSIONS}

The excellent agreement between measurement and calculation of the microwave beam profile within the resolution of $15^{\circ}$ demonstrates the reliability of the operation of the measurement technique. This device allows to map transmission lines where polarizer plates with unknown characteristics are included. It also allows to map the beam characteristics if transmission lines are used for various frequencies. The device was made for frequencies around $110 \mathrm{GHz}$, but the method can, in principle, be used for any frequency in the millimeter wave range.

\section{ACKNOWLEDGMENTS}

The authors would like to thank Dr. Paul Woskov (PSFC, MIT) for discussion and the FOM group at the TEXTOR-tokamak experiment for supporting these experiments at the gyrotron box $(110 \mathrm{GHz})$.

${ }^{1}$ J. Wesson, Tokamaks, 2nd ed. (Oxford, New York, 1997).

${ }^{2}$ M. Thumm, Plasma Phys. Controlled Fusion 45, A143 (2003).

${ }^{3}$ M. Thumm and W. Kasparek, IEEE Trans. Plasma Sci. 30, 755 (2002).

${ }^{4}$ D. Wagner and F. Leuterer, Int. J. Infrared Millim. Waves 26, 163 (2005).

${ }^{5}$ Y. L. Kok and N. C. Gallagher, J. Opt. Soc. Am. A 5, 65 (1988).

${ }^{6}$ H. Bindslev, J. Atmos. Terr. Phys. 58, 983 (1996).

${ }^{7}$ H. Bindslev, S. K. Nielsen, L. Porte, J. A. Hoekzema, S. B. Korsholm, F. Meo, P. K. Michelsen, S. Michelsen, J. W. Oosterbeek, E. L. Tsakadze, E. Westerhof, P. Woskov, and the TEXTOR team, Phys. Rev. Lett. 97, 205005 (2006).

${ }^{8}$ M. Born and E. Wolf, Principles of Optics (Pergamon, Oxford, 1965), pp. 25-30.

${ }^{9}$ R. M. A. Azzam and N. M. Bashara, Ellipsometry and Polarized Light (North-Holland, Amsterdam, 1977).

${ }^{10}$ M. V. Klein and T. E. Furtak, Optik (Springer, Berlin, 1988).

${ }^{11}$ H. Ikezi, C. P. Moeller, J. L. Doane, M. DiMartino, J. Lohr, D. Ponce, and R. W. Callis, Rev. Sci. Instrum. 70, 1994 (1999).

${ }^{12}$ T. Notake, H. Idei, S. Kubo, T. Shimozuma, Y. Yoshimura, S. Kobayashi, Y. Mizuno, S. Ito, Y. Takita, K. Ohkubo, W. Kasparek, T. Watari, and R. Kumazawa, Rev. Sci. Instrum. 76, 023504 (2005).

${ }^{13}$ H. J. Hartfuss, T. Geist, and M. Hirsch, Plasma Phys. Controlled Fusion 39, 1693 (1997)

${ }^{14}$ F. Leipold, R. H. Stark, A. El-Habachi, and K. H. Schoenbach, J. Phys. D: Appl. Phys. 33, 2268 (2000).

${ }^{15}$ N. J. Dobbe, A. B. Sterk, R. P. J. J. M. Kruisbergen, O. G. Kruyt, M. E. Bestbreurtje, P. R. Prins, J. A. Hoekzema, A. F. van der Grift, and B. S. Q. Elzendoorn, Fusion Eng. Des. 56, 599 (2001).

${ }^{16}$ E. Westerhof, J. A. Hoekzema, G. M. D. Hogeweij, R. J. E. Jaspers, F. C. Schuller, C. J. Barth, W. A. Bongers, A. J. H. Donne, P. Dumortier, A. F. van der Grift, J. C. van Gorkom, D. Kalupin, H. R. Koslowski, A. KramerFlecken, O. G. Kruijt, N. J. L. Cardozo, P. Mantica, H. J. van der Meiden, A. Merkulov, A. Messiaen, J. W. Oosterbeek, T. Oyevaar, A. J. Poelman, R. W. Polman, P. R. Prins, J. Scholten, A. B. Sterk, C. J. Tito, V. S. Udintsev, B. Unterberg, M. Vervier, and G. van Wassenhove, Nucl. Fusion 43, 1371 (2003) 